\title{
GSE says goodbye to paper!
}

You are looking at the last "paper" version of GSE mailed to our subscribers. Indeed, as explained in the previous issue (volume 40, issue 5), Genetics Selection Evolution (GSE) is moving to "Open Access" publishing from 1st January 2009.

With this last "paper" editorial, we wish to thank all our subscribers, readers, authors, editors and reviewers who have supported GSE and have actively participated in its development in the last 40 years.

Saying goodbye to the paper version of GSE may represent a small step towards being more environmentally friendly. This is not the main issue but rather our goal is to keep pace with the major changes in publishing practices and the circulation of scientific information.

Our readers will notice little change in the journal's content but beginning with volume 41 in year 2009, they will have free, immediate, permanent and full-text online access to all GSE's past and new peer-reviewed research publications.

Inevitably, there will be a period of transition between the current subscription-based system and the coming author-payment model. All our editorial and management staff will strive to keep things simple and transparent. From November 2008, a provisional webpage will be opened at http://www.genet-sel-evol.org/ to provide all the necessary information.

We are happy to announce that from January 2009, at least a dozen highly interesting papers will be released in the new "Open Access" GSE journal dedicated to research on genetics and selection in farm and experimental animals.

So it is farewell and not goodbye! 